\title{
Small electric and magnetic signals observed before the arrival of seismic wave
}

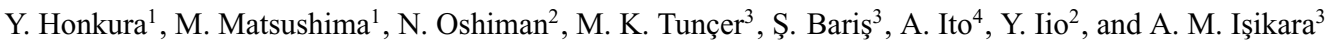 \\ ${ }^{1}$ Department of Earth and Planetary Sciences, Tokyo Institute of Technology, Tokyo 152-8551, Japan \\ ${ }^{2}$ Disaster Prevention Research Institute, Kyoto University, Kyoto 611-0011, Japan \\ ${ }^{3}$ Kandilli Observatory and Earthquake Research Institute, Boğaziçi University, Istanbul 81220, Turkey \\ ${ }^{4}$ Faculty of Education, Utsunomiya University, Utsunomiya 321-8505, Japan
}

(Received September 10, 2002; Revised November 14, 2002; Accepted December 6, 2002)

\begin{abstract}
Electric and magnetic data were obtained above the focal area in association with the 1999 Izmit, Turkey earthquake. The acquired data are extremely important for studies of electromagnetic phenomena associated with earthquakes, which have attracted much attention even without clear physical understanding of their characteristics. We have already reported that large electric and magnetic variations observed during the earthquake were simply due to seismic waves through the mechanism of seismic dynamo effect, because they appeared neither before nor simultaneously with the origin time of the earthquake but a few seconds later, with the arrival of seismic wave. In this letter we show the result of our further analyses. Our detailed examination of the electric and magnetic data disclosed small signals appearing less than one second before the large signals associated with the seismic waves. It is not yet solved whether this observational fact is simply one aspect of the seismic dynamo effect or requires a new mechanism.
\end{abstract}

Key words: Izmit earthquake, seismic dynamo effect, seismic wave, electric and magnetic changes

\section{Introduction}

Observations of changes in the electromagnetic field before earthquake occurrences have been supposed as one of the possible methods in earthquake prediction (e.g. Honkura, 1981). However, no firm observational evidence with a clear physical mechanism has been obtained, although some likely mechanisms have been proposed to account for ambiguous observational results; the electrokinetic effect (Mizutani et al., 1976; Ishido and Mizutani, 1981; Gershenzon et al., 1993; Haartsen and Pride, 1997), the piezoelectric effect (Gershenzon et al., 1993), the piezomagnetic effect (Stacey and Johnston, 1972; Sasai, 1980), and the electromagnetic induction effect (Gershenzon et al., 1993; Iyemori et al., 1996; Honkura et al., 2000; Matsushima et al., 2002).

Recent examples of unambiguous electric field changes indicate that the arrival of electric signals is synchronized with the arrival of seismic waves (Yamada and Murakami, 1982; Mogi et al., 2000; Nagao et al., 2000), but the possibility was pointed out that the magnetic field started to change before seismic wave arrival in the case of the 1995 Kobe earthquake (Iyemori et al., 1996). In the meantime, we could obtain a set of electromagnetic data in the focal area of the 1999 Izmit earthquake (Honkura et al., 2000; Matsushima et $a l ., 2002)$, which is extremely valuable for studies of electromagnetic field changes associated with earthquakes. Some overall characteristics of electric and magnetic field changes were already shown (Honkura et al., 2000; Matsushima et al., 2002), and in this letter we focus our attention on the new finding.

Copy right(c) The Society of Geomagnetism and Earth, Planetary and Space Sciences (SGEPSS); The Seismological Society of Japan; The Volcanological Society of Japan; The Geodetic Society of Japan; The Japanese Society for Planetary Sciences.

\section{Data}

The data were obtained at four sites, denoted by 118, 120, 121 and 122 in Fig. 1, among many sites temporarily established for magnetotelluric (MT) observations across two fault zones; the northern one was in fact ruptured at the time of the Izmit earthquake. Also at the reference site, denoted by 001 in Fig. 1, MT signals were measured for remote reference processing of MT data. Hence the simultaneous electric (two components) and magnetic (three components) data at five sites are available.

\section{On the Arrival Time of Electric and Magnetic Signals}

We first try to identify the arrival time of electric and magnetic signals in the raw time series data at four sites located above the focal area. The electric and magnetic signals seem to have arrived about three seconds after the origin time of the Izmit earthquake as shown in Fig. 2, and no significant changes can be seen before and during a few seconds after the origin time. The arrival time of these electric and magnetic signals seems to correspond to the arrival time of seismic waves, but we cannot confirm this because no seismic records are available at the MT sites.

In this respect, we can use another set of electromagnetic and seismic velocity records simultaneously acquired at sites 121 and 122 during aftershock activity. In this case, however, the electromagnetic signals for small aftershocks are so small that they are obscured by naturally occurring MT variations, which obviously have nothing to do with earthquakes. Hence it is not straightforward to identify the arrival time of electromagnetic signals. 


\section{Removal of Known MT Signals}

Natural MT signals consist of external and internal origins, the latter resulting from electromagnetic induction in the conducting Earth. The scale length of MT signals of external origin is so long that they can be regarded as uniform over the region shown in Fig. 1. Also the electromagnetic induction is governed by a set of linear equations. Hence, each component of MT fields at sites 118, 120, 121 and 122 should be correlated with the horizontal magnetic field at the reference site 001 ; in fact, this is the basis for remote refer-

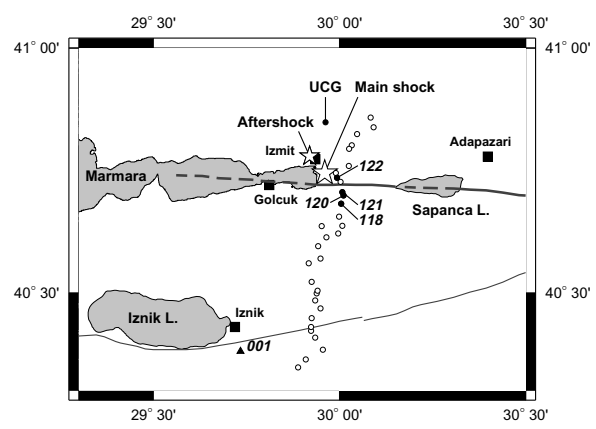

Fig. 1. Locations of magnetotelluric (MT) observation sites along a north-south profile crossing the focal area of the 1999 Izmit earthquake (Honkura et al., 2000). At the sites denoted by 118, 120, 121 and 122 , MT measurements were in operation during the Izmit earthquake in addition to the reference site denoted by 001 near the southern fault. A large star symbol shows the epicenter of the Izmit earthquake (Honkura et al., 2000). A small star symbol located near Izmit City indicates the epicenter of a small aftershock for which the electromagnetic and seismic records are shown in Fig. 3. UCG is the closest seismic station operated by TÜBITAK (Özalaybey et al., 2002). ence processing in MT. We can therefore predict MT signals at sites 118, 120, 121 and 122 from the northward and the eastward magnetic field variations at the reference site 001 , which are regarded as inputs to a kind of linear system. In the present case, we used the multi-channel Wiener filtering technique (Davis et al., 1981), and found that it is effective, particularly for the magnetic field, in removing the known, predictable MT signals from the observed data at four sites.

\section{Electric and Magnetic Signals Associated with an Aftershock}

One example for an aftershock located beneath Izmit City (see Fig. 1) is shown in Fig. 3. At site 121, small yet clear signals can be distinguished from the background noise after the prediction operation. In this case, the onset of electric and magnetic field changes seems to be simultaneous with the P-wave arrival within the accuracy of $1 / 24$ second (MT data sampling interval). At site 122, only magnetic signals can be identified within the time interval shown in the figure, and the onset in the northward $(H x)$ and eastward $(H y)$ components of the magnetic field is slightly later than the P-wave arrival.

As for the mechanism of such electric and magnetic signals, we have proposed the generation of electric and magnetic fields by seismic waves (Honkura et al., 2000; Matsushima et al., 2002). It is well known that motion of a conductor in the magnetic field gives rise to an electromotive force within the conductor. Seismic waves are obviously regarded as oscillations of the elastic and conducting Earth, and the Earth's magnetic field of core origin prevails everywhere in the Earth. We have called this mechanism the 'seismic dynamo effect', referring to the core dynamo originating (a) Site 118

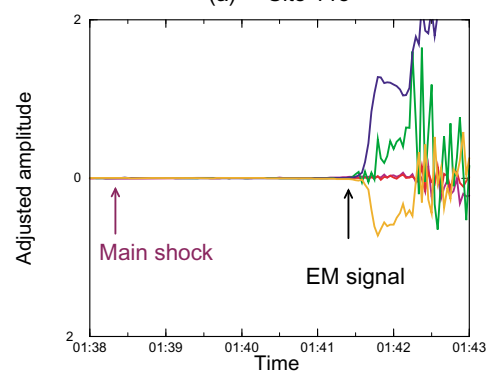

(b) Site 120

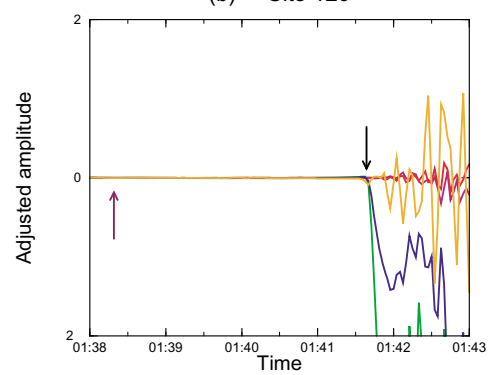

(c) Site 121

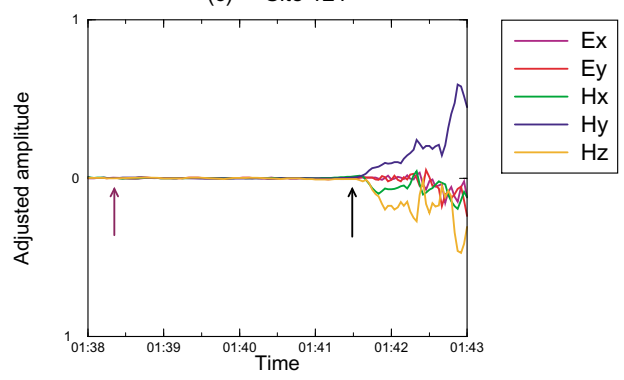

(d) Site 122

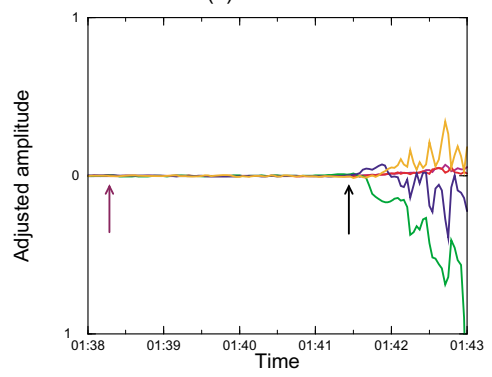

Fig. 2. Electric and magnetic field variations at (a) site 118, (b) site 120, (c) site 121 and (d) site 122 . Ex and Ey are the northward and the eastward electric fields, respectively. $\mathrm{Hx}, \mathrm{Hy}$ and $\mathrm{Hz}$ are the northward, the eastward and the downward magnetic fields. The amplitudes are adjusted so that the noise level before the onset of changes indicated by a black arrow becomes nearly the same for all the components at each site. The origin of the abscissa corresponds to $00 \mathrm{~h} 01 \mathrm{~m} 38 \mathrm{~s}$ (UTC), August 17, 1999. The origin time of the Izmit earthquake is $00 \mathrm{~h} 01 \mathrm{~m} 38.37 \mathrm{~s}$ (Honkura et al., 2000) as indicated by another arrow. The arrival of electromagnetic field signals has been interpreted as corresponding to the first arrival of seismic wave, before which no changes exceeding the noise level can be seen in the figure. 
(a) Electric field at site 121

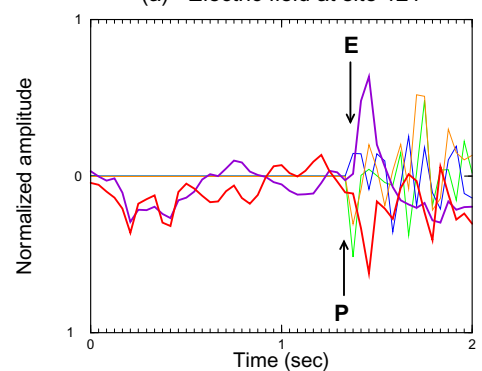

(b) Magnetic field at site 121

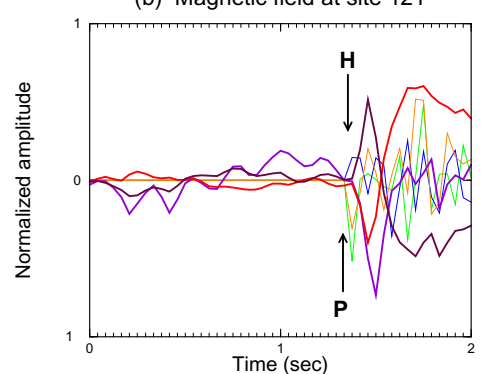

(c) Electric field at site 122

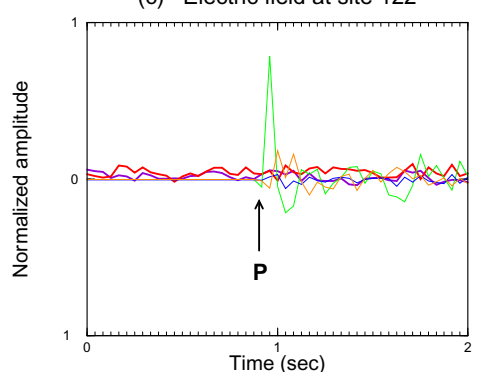

(d) Magnetic field at site 122

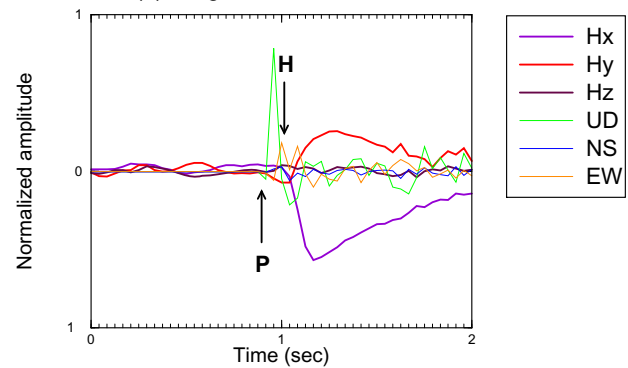

Fig. 3. Electric and magnetic field variations associated with the aftershock shown in Fig. 1. Ex and Ey are the northward and the eastward electric fields, respectively. UD, NS and EW are the vertical, the north-south and the east-west components of ground velocity. $H x, H y$ and $H z$ are the northward, the eastward and the downward magnetic fields. The amplitude is normalized for each component with respect to its maximum range in a later main portion showing larger variations. As in Fig. 2, the noise level before the onset of changes associated with seismic waves is nearly the same for all the components at each site. The origin of the abscissa corresponds to $22 \mathrm{~h} 13 \mathrm{~m} 31.25 \mathrm{~s}$ (UTC), September 18, 1999. The arrows with E and H indicate the estimated arrival time of electric and magnetic signals, respectively. The arrow with P denotes the arrival time of seismic P-wave as clearly identified by the vertical component. The sampling frequency is $24 \mathrm{~Hz}$ and hence the time resolution is $1 / 24$ second as indicated by ticks on the abscissa. In this example, the arrival of electromagnetic fields can be regarded as simultaneous with the P-wave arrival.

(a) Site 118

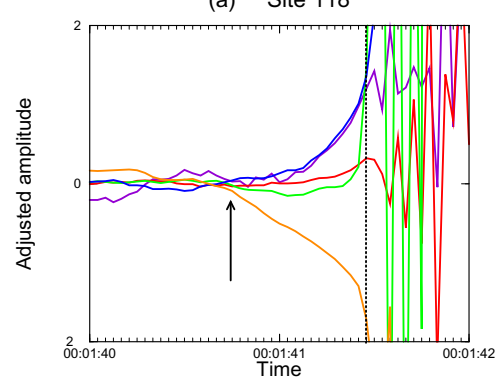

(b) Site 120

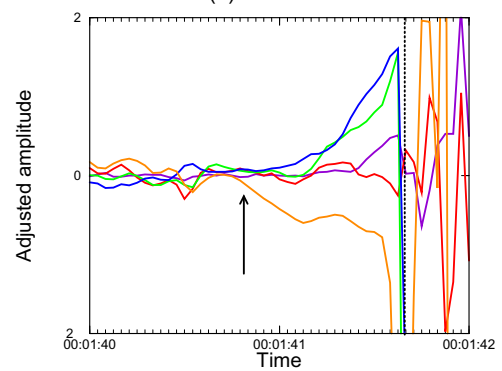

(c) Site 121

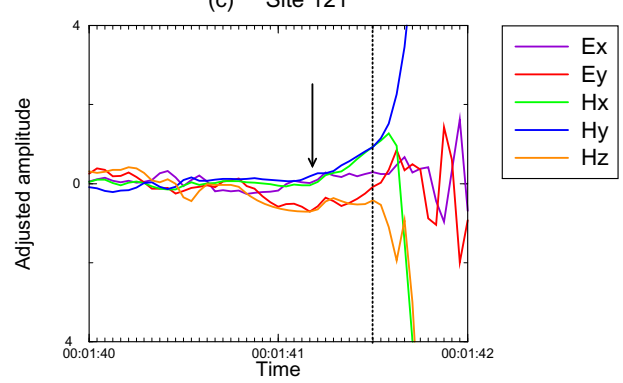

(d) Site 122

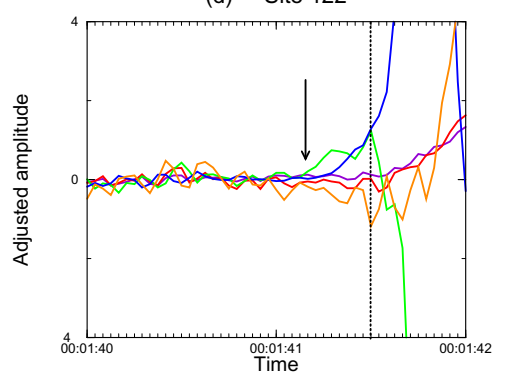

Fig. 4. Close-up view of Fig. 2; variations are made two orders of magnitude larger. The origin of the abscissa corresponds to $00 \mathrm{~h} 01 \mathrm{~m} 40 \mathrm{~s}$ (UTC), August 17, 1999. The scale of this figure is nearly the same as that of Fig. 3, as understood by the noise levels before the signals appear. The arrival of electromagnetic signals corresponding to the first arrival of seismic wave is indicated by a vertical dotted line. We can recognize slow rises in some components before the dotted lines, but the arrival times, as indicated by arrows, of these slow signals are earlier only by a fraction of second at all the sites. Nonetheless, these signals exceed the noise levels and hence they are significant.

from the term $\boldsymbol{v} \times \boldsymbol{B}$ where $\boldsymbol{v}$ is the velocity of conducting fluid in the Earth's core and $\boldsymbol{B}$ is the magnetic field. The only difference is that in the seismic dynamo effect $v$ is the motion of elastic medium, instead of fluid motion. For quantitative analyses we must certainly take into account the effect of vibrations of the MT equipment. 


\section{Electric and Magnetic Signals Associated with the Main Shock}

In the case of a small aftershock as shown in Fig. 3, the electromagnetic signals are slightly larger than the noise level, but the signals for the main shock shown in Fig. 2 are much larger and we can examine the signals in more detail. Here we focus our attention on the portions near the arrival of electromagnetic signals. Surprisingly, as shown in Fig. 4, gradual changes in some components $(E x, H y, H z$ at site 118; $\mathrm{Hx}, \mathrm{Hy}, \mathrm{Hz}$ at site $120 ; \mathrm{Hx}, \mathrm{Hy}$ at site $121 ; \mathrm{Hx}, \mathrm{Hy}$ at site 122) can be seen before the expected arrival of seismic waves. Duration of such changes is only a fraction of one second and their arrival is well after the origin time of the Izmit earthquake.

\section{Discussion}

The reason why such earlier arrival of electromagnetic signals could not be detected in the case of the small aftershock shown in Fig. 3 is simply because the signals themselves are much smaller, as explained in more detail below. In Figs. 2 and 4 , the ordinate scales are adjusted, respectively, so that the signals under consideration are well recognized. In fact, the scale of the ordinate of Fig. 4 is magnified by two orders of magnitude, compared with Fig. 2, and it is now comparable with that of Fig. 3, in which the amplitude of electric field variation is the order of $10^{-6}-10^{-7} \mathrm{~V} / \mathrm{m}$. For the magnetic field, we must make corrections of the frequency-dependent sensor response to the output data and in the case of Fig. 3, the amplitude of magnetic field variation turned out to be of the order of $10^{-12} \mathrm{~T}$. The signals for the main shock $(\mathrm{Ms}=$ 7.4) are at least two orders of magnitude larger than those for the aftershock $\left(\mathrm{M}_{\mathrm{L}}=2.8\right)$.

Theoretically, one may claim that electromagnetic signals should propagate in the Earth with the electromagnetic-wave speed and hence they should be observed much earlier than the arrival of seismic waves, nearly simultaneously with the origin time of the main shock. We have clearly shown that this is not the case, observationally. The pieces of evidence shown in this letter impose firm constraints on the mechanism of propagation of electromagnetic signals, including attenuation of the high-frequency signals in the conducting Earth (Honkura and Kuwata, 1993). In particular, this letter stimulates us to a challenging theoretical work on the seismic dynamo effect; electromagnetic induction in the conducting Earth by a traveling electromotive source due to seismic waves radiated from the earthquake source area.

Alternatively, such an earlier signal may be related to a possible initial slow slip immediately before the main fault rupture (e.g. Iio, 1995). However, as pointed out by Johnston and Linde (2002), such a slow slip is at least three orders of magnitude smaller than the slip during the main shock. Our results show that the slow electromagnetic variations are only two orders of magnitude smaller than those during the main shock and hence they are unlikely to be associated with a slow slip. The seismic record at UCG (see Fig. 1) also shows no event corresponding to the slow electromagnetic variations.

Acknowledgments. The precious set of data could be acquired during a difficult condition after the Izmit earthquake with the help of many colleagues at Boğaziçi University. We thank them for their great contribution to the hard work in the field. We also thank Serdar Özalaybey for the seismic data at UCG and the referees for useful comments. This work was supported by the Ministry of Education, Science, Culture and Sports of Japan under grant-in-aid for scientific research Nos. 11694063 and 13373002, and also by the research fund of Boğaziçi University.

\section{References}

Davis, P. M., D. D. Jackson, C. A. Searls, and R. L. McPherron, Detection of tectonomagnetic events using multichannel predictive filtering, $J$. Geophys. Res., 86, 1731-1737, 1981.

Gershenzon, N. I., M. B. Gokhberg, and S. L. Yunga, On the electromagnetic field of an earthquake focus, Phys. Earth Planet. Inter., 77, 13-19, 1993.

Haartsen, M. W. and S. R. Pride, Electroseismic waves from point sources in layered media, J. Geophys. Res., 102, 24745-24769, 1997.

Honkura, Y., Electric and magnetic approach to earthquake prediction, in Current Research in Earthquake Prediction I, Edited by T. Rikitake, pp. 301-383, Center Academic Publications Japan/ D. Reidel Publishing Company, 1981.

Honkura, Y. and Y. Kuwata, Estimation of electric fields in the conducting Earth's crust for oscillating electric current dipole sources and implications for anomalous electric fields associated with earthquakes, Tectonophysics, 224, 257-263, 1993.

Honkura, Y., A. M. Işikara, N. Oshiman, A. Ito, B. Üçer, Ş. Bariş, M. K Tunçer, M. Matsushima, R. Pektaş, C. Çelik, S. B. Tank, F. Takahashi, M. Nakanishi, R. Yoshimura, Y. Ikeda, and T. Komut, Preliminary results of multidisciplinary observations before, during and after the Kocaeli (Izmit) earthquake in the western part of the North Anatolian Fault Zone, Earth Planets Space, 52, 293-298, 2000.

Iio, Y., Observations of the slow initial phase generated by microearthquakes: implications for earthquake nucleation and propagation, J. Geophys. Res., 100, 15333-15349, 1995.

Ishido, T. and H. Mizutani, Experimental and theoretical basis of electrokinetic phenomena in rock-water systems and its application to geophysics, J. Geophys. Res., 86, 1763-1775, 1981.

Iyemori, T., T. Kamei, Y. Tanaka, M. Takeda, T. Hashimoto, T. Araki, T. Okamoto, K. Watanabe, N. Sumitomo, and N. Oshiman, Co-seismic geomagnetic variations observed at the 1995 Hyogoken-nanbu earthquake, J. Geomag. Geoelectr., 48, 1059-1070, 1996.

Johnston, M. J. S. and A. T. Linde, Implications of crustal strain during conventional, slow, and silent earthquakes, in International Handbook of Earthquake and Engineering Seismology, 81A, pp. 589-605, 2002.

Matsushima, M., Y. Honkura, N. Oshiman, Ș. Bariș, M. K. Tunçer, S. B Tank, C. Çerik, F. Takahashi, M. Nakanishi, R. Yoshimura, R. Pektaş, T. Komut, E. Tolak, A. Ito, Y. Iio, and A. M. Işikara, Seimo-elecromagnetic effect associated with the Izmit earthquake and its aftershocks, Bull. Seismol. Soc. Am., 92, 350-360, 2002.

Mizutani, H., T. Ishido, T. Yokokura, and S. Ohnishi, Electrokinetic phenomena associated with earthquakes, Geophys. Res. Lett., 3, 365-368, 1976.

Mogi, T., Y. Tanaka, D. S. Widarto, E. M. Arsadi, N. T. Puspito, T. Nagao, W. Kanda, and S. Uyeda, Geoelectric potential difference monitoring in southern Sumatra, Indonesia - Co-seismic change-, Earth Planets Space, 52, 245-252, 2000.

Nagao, T., Y. Orihara, T. Yamaguchi, I. Takahashi, K. Hattori, Y. Noda, K. Sayanagi, and S. Uyeda, Co-seismic geoelectric potential changes observed in Japan, Geophys. Res. Lett., 27, 1535-1538, 2000.

Özalaybey, S., M. Ergin, M. Aktar, C. Tapirdamaz, F. Biçmen, and A. Yörük, The 1999 Izmit earthquake sequence in Turkey: seismological and tectonic aspects, Bull. Seismol. Soc. Am., 92, 376-386, 2002.

Sasai, Y., Application of the elasticity theory of dislocation to tectonomagnetic modeling, Bull. Earthq. Res. Inst., 55, 387-447, 1980.

Stacey, F. D. and M. J. S. Johnston, Theory of the piezo-magnetic effect in titanomagentite-bearing rocks, Pure Appl. Geophys., 97, 146-155, 1972.

Yamada, I. and H. Murakami, Self-potential variations associated with quarry blasts, Zisin, 35, 393-400, 1982 (in Japanese with English abstract)

Y. Honkura (e-mail: yhonkura@geo.titech.ac.jp), M. Matsushima, N Oshiman, M. K. Tunçer, Ş. Bariş, A. Ito, Y. Iio, A. M. Işikara 